\title{
Design and Manufacturing of a High-Precision Sun Tracking System Based on Image Processing
}

\author{
Kianoosh Azizi and Ali Ghaffari \\ Faculty of Mechanical Engineering, K. N. Toosi University of Technology, Pardis Avenue, Molla-Sadra Avenue, Vanak Sq., \\ P.O. Box 19395-1999, Tehran 19991 43344, Iran \\ Correspondence should be addressed to Kianoosh Azizi; kianooshazizi@gmail.com
}

Received 30 May 2013; Revised 5 August 2013; Accepted 5 August 2013

Academic Editor: Mohammad A. Behnajady

Copyright ( $\odot 2013$ K. Azizi and A. Ghaffari. This is an open access article distributed under the Creative Commons Attribution License, which permits unrestricted use, distribution, and reproduction in any medium, provided the original work is properly cited.

\begin{abstract}
Concentration solar arrays require greater solar tracking precision than conventional photovoltaic arrays. This paper presents a high precision low cost dual axis sun tracking system based on image processing for concentration photovoltaic applications. An imaging device is designed according to the principle of pinhole imaging, making sun rays to be received on a screen through pinhole and to be a sun spot. The location of the spot is used to adjust the orientation of the solar panel. A fuzzy logic controller is developed to achieve this goal. A prototype was built, and experimental results have proven the good performance of the proposed system and low error of tracking. The operation of this system is independent of geographical location, initial calibration, and periodical regulations.
\end{abstract}

\section{Introduction}

Renewable energies are considered as a great source of energy during the last two decades. As an important source of alternative energy, the solar has unlimited reserves, has widespread existence, is pollution-free, and so forth. It is shown that optimum of solar energy is obtained when sun rays are incident normally on the transforming part of solar systems, such as solar thermal collectors, solar cells, and other solar equipments. Among various types of solar cells, the application of high concentration solar cells allows a significant increase in the amount of energy collected by solar arrays per area unit. However, the performance of solar cells with concentrators decreases drastically if the sun pointing error is greater than a small value and, therefore, a low tracking error must be achieved for this kind of solar photovoltaic arrays (PV) compared with conventional PV arrays [1]. Several kind of solar tracking systems are proposed in the literature; one can classify them according to their degrees of freedom (DoFs) and/or control strategy. Regarding DoF, there are three main types of trackers [2]: fixed devices, single-axis trackers (see [3]), and dual-axis trackers (see [4]). It is shown that the annual gain in energy production of the dual and single-axis trackers is 1.5 and $1.40-1.45$, respectively, compared with the nontracking systems [1]. Regarding control strategy, three main types of solar trackers exist [5]: passive, open-loop, and closed-loop controlled trackers. The passive trackers have no electronic sensors or actuators [6]. The open-loop ones have no sensors too but use microprocessor and are based on mathematical formulae to predict the sun's position $[7,8]$. The third kind of trackers use the information of electro-optic sensors for estimating the location of the sun $[5,9]$. The open-loop trackers are dependent on geographical location and the start time situation of the system, while closed-loop systems do not have these disadvantages.

An open-loop type of controller does not observe the output of the processes that it is controlling. Consequently, an open-loop system cannot correct any errors so that it could make and may not compensate for disturbances in the system. The system is simpler and cheaper than the closedloop type of sun tracking systems [10]. In open-loop mode, the computer or a processor calculates the sun's position from formulae or algorithms using its time/date and geographical information to send signals to the electromotor. However, in some cases many sensors are used to identify specific positions [11]. The systems proposed in [12,13] are in this category. 
In closed-loop systems, a number of inputs are transferred to a controller from sensors which detect relevant parameters induced by the sun, manipulated in the controller and then yield outputs [10]. In this type, by differential illumination of electrooptical sensors differential control signal occurs which is used to drive the motor and to orient the apparatus in such direction where illumination of electrooptical sensors becomes equal and balanced. In addition, the photodiodes can be mounted on tilted planes in order to increase the photocurrent sensitivity, and, very commonly in concentrator PV applications, the shading device is presented as a collimating tube which prevents diffuse irradiation from entering the sensor and masking a precise measurement of the sun alignment position [14]. Such trackers, with high accuracy, are intended mainly for concentrator solar systems. These trackers are complex and, therefore, expensive and also unreliable [11]. Examples of these systems are proposed in [15-17]. More comparison between various types of sun tracking systems can be achieved in review articles.

The target of this study is to design and manufacture a low cost and precise sun tracking system for concentration photovoltaic panels (CPVs). The provided sun tracking systems in the recent studies are applicable for PV panels not for CPVs. Anyway they have their weaknesses that are listed below.

(i) Tracking precision of low cost systems is not mentioned in most studies,

(ii) Most of these tracking systems are inappropriate for CPV because of their low precision.

(iii) The precise systems (for instance manufactured by Kipp \& Zonen Company) are relatively expensive.

According to the literature, in most low cost sun trackers, the discrete elements, light dependent resistors (LDRs), are used as sensor. In this method of sun tracking, the differences between the outputs of at least four LDRs are used to generate error signals in two directions of tracking. In these systems, for instance, when the outputs of eastern and western LDRs become equal, the east to west tracking ends. But when the outputs of the LDRs are the same, the tracking error is actually not zero. This LDR-based tracking error is not acceptable for CPV applications. This error exists because that all four sensors are not exactly the same, and the second reason is the influences of weather condition on this type of sensors. So this kind of sensors is not appropriate for CPV applications.

The solar trackers have nonlinear behavior and are subjected to disturbances. For such systems it is recommended to apply soft computing methods to control them. In recent years, fuzzy logic has become an important approach in designing nonlinear controllers because of its simplicity, ease of design and implementation [18].

In this paper a high-precision and low cost dual-axis sun tracker (DAST) is proposed for PV applications. To achieve optimal solar tracking, a fuzzy algorithm is developed. At first, the orientation of the solar panel is approximately adjusted using four LDRs, and then the strategy based on image processing decreases tracking error, as much possible as.

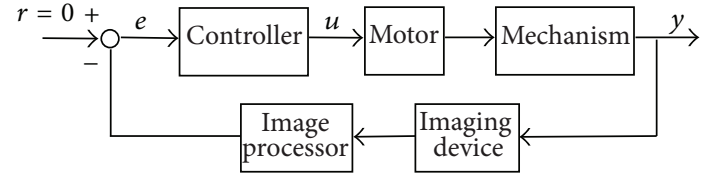

FIgURE 1: Closed loop control system.

In other words, adding camera and image processing-based method strongly diminished the tracking errors that are provided in LDR-based tracking methods. This system does not need any astronomical consideration in finding the sun location and is independent of geographical position and initial configuration.

The remainder of the paper is organized in four sections as follows. in Section 2 the fundamental operation of the system is explained. The overall system and control strategy are described in detail in Section 3. Experimental results are shown in Section 4, and finally, in Section 5 the conclusions of this work are drawn.

\section{Principle of the System Operation}

There are two noticeable motions for the sun in respect to an observer on the earth. One is called azimuthal motion (from east to west), and the other is called altitudinal motion (refering to the changing of the height of sun in the sky). Therefore, it is necessary to consider two degrees of freedom for motions of any solar tracker power generator. The proposed system in this work mainly consists of the imaging device, image acquisition and processing parts, controller, and electromechanical structure. The working principle is shown in Figure 1.

The tracking algorithm has two modes: LDR-based mode and image processing-based mode. LDR is a discrete element. One of the disadvantages of using this type of sensor is its high sensitivity to weather conditions such as temperature and humidity. To overcome this disadvantage and to provide less tracking error, the tracking algorithm based on image processing has been proposed. The imaging device is shown in Figure 2. It consists of a box, a transparent screen in the middle, and a camera on the bottom. A suitable small hole is opened on the middle of the top of the box. Sun ray can only be entered from this hole. A glass lid is set on the top of the box to protect transparent screen and imaging device against penetration of the rain and dust. According to the principle of pinhole imaging, the sun rays entered to the chamber form a beam to produce a small bright spot on the receiving screen (Figure 3(a)). The transparent screen is assumed on the XY surface as shown in Figure 3(b). Now at a moment, the sun spot on the transparent screen has a coordinates $(x, y)$. Since the receiving screen is parallel to the surface of the solar panel, the target coordinates for sun spot are $(0,0)$.

After image acquisition, the position of the sun spot should be determined through image processing. Then, error signal is fed to fuzzy logic controller (FLC) and suitable control signals are emitted to motors. This procedure is repeated till tracking error is reached to desired range. 


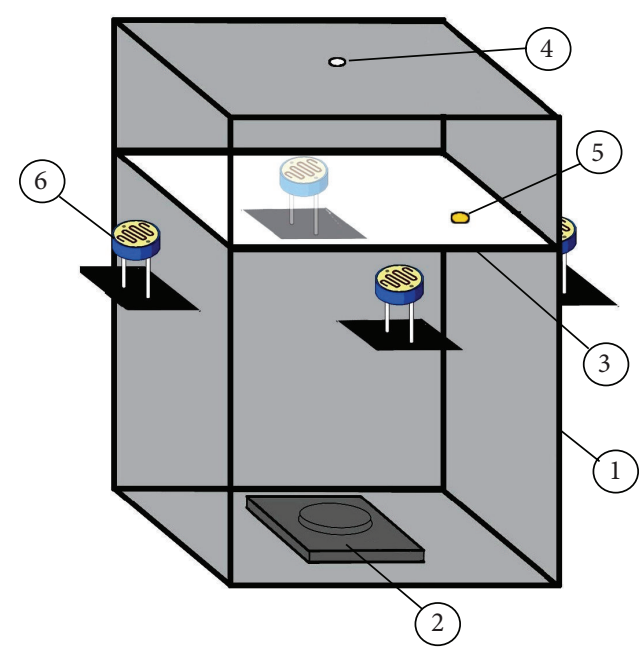

FIGURE 2: Imaging device and LDR (casing (1), webcam (2), transparent screen (3), pinhole (4), sunspot (5), and LDR (6)).

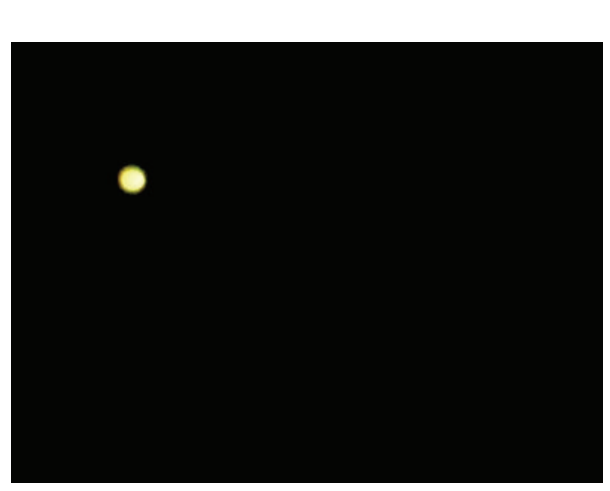

(a)

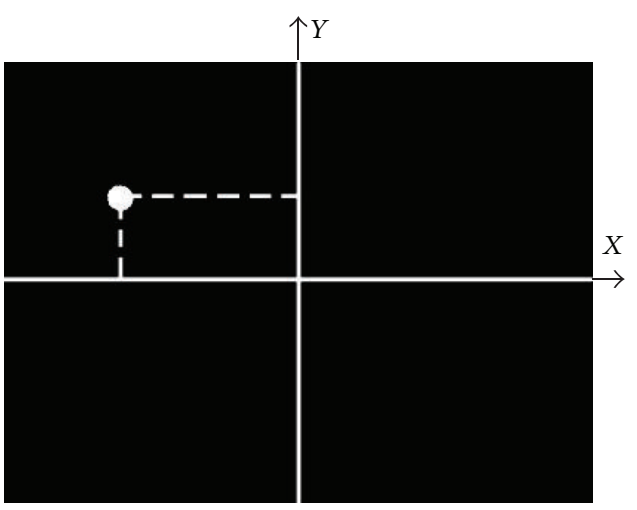

(b)

FIgURE 3: Acquisited (a) and processed (b) image.

\section{System Description}

3.1. Dual-Axis Electromechanical Structure. Electromechanical structure has two degrees of freedom, motorized by two DC motors: a base platform moving around vertical axis and a suspended platform with PV panel moving around horizontal axis. Position of the base platform and the suspended frame can vary in the range of $\pm 90^{\circ}$ ensuring alignment of the panel in azimuth and elevation, respectively. Several components of this structure such as motors, gearboxes, and thrust ball bearing are shown in Figure 4.

\subsection{Image Acquisition and Processing Unit. An A4TECH} (PK-836F) commercial webcam that offers $640 * 480$ pixels was used. A polarized filter is set under the pinhole, in the box, to prevent saturation of the charge-coupled device of the camera in intensive solar radiations. A diaphragm of calk paper is used as the transparent screen. The webcam was connected to a personal computer. A simple MATLAB program was used to image processing. This processing algorithm calculates the coordinate $(x, y)$ of the center of the sun spot.

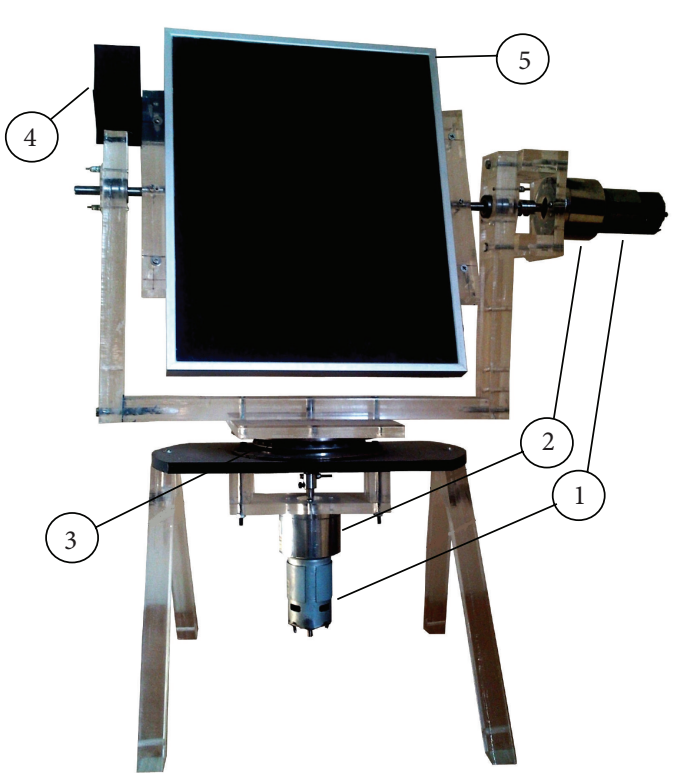

FIGURE 4: Electromechanical structure (motors (1), gearboxes (2), thrust bearing (3), imaging device (4), and solar panel (5)). 


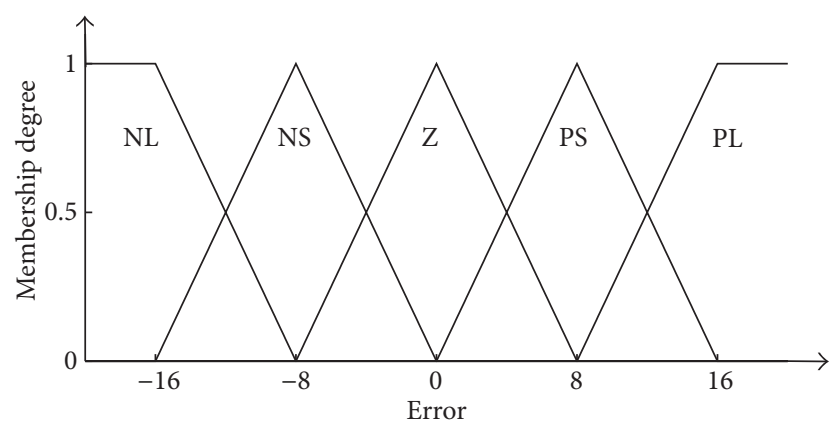

FIGURE 5: Input membership functions of the FLC.

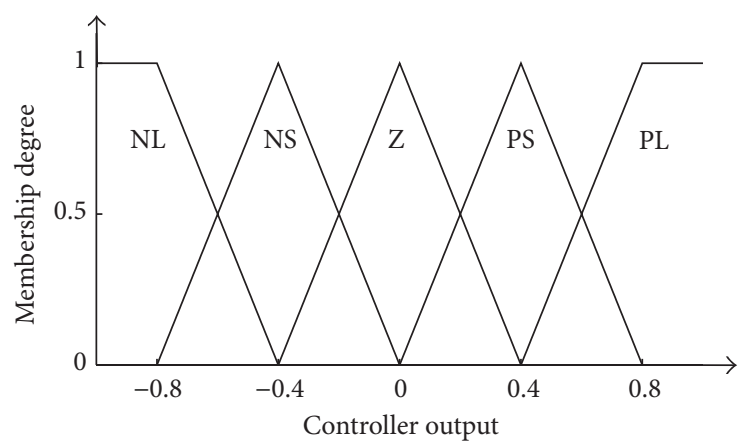

FIgURE 6: Output membership function of the FLC.

3.3. Control Unit. The fuzzy logic can make human knowledge into the knowledgebase to control a plant with linguistic descriptions. The advantages of FLC including good popularization and high faults tolerance make it suitable for nonlinear control systems. A FLC has five parts: fuzzifier, data base, rule base, inference mechanism, and defuzzifier.

At first, the sun lights radiate on four LDRs, and the differences of the voltage on the eastern-western LDRs and the southern-northern LDRs will be delivered into the controller; then motors actuate till the differences of these sensors voltage become zero. Then the image processing-based mode will operate. After calculation of the coordinates of the sun spot, $x$ and $y$ are fed to controller as error signals $(e)$. When the coordinates of the sun spot are being $(0,0)$, the controller does not work. Since the sun moves very slow, the fast rotating speed of the solar tracking device is not necessary. By fuzzy control, some advantages such as reducing consumption power of motors and fast and smooth positioning can be achieved. Control of motors may be done independently. The rotation angel of motor is considered as the output variable of fuzzy controller $(u)$. The membership functions are shown in Figures 5 and 6 . Five fuzzy control rules are used, as shown in the following:

R1: If $e$ is positive large (PL), then $u$ is $\mathrm{PL}$,

$\mathrm{R} 2$ : If $e$ is positive small (PS), then $u$ is PS,

R3: If $e$ is zero $(\mathrm{Z})$, then $u$ is $\mathrm{Z}$,

R4: If $e$ is negative small (NS), then $u$ is NS,

R5: If $e$ is negative large (NL), then $u$ is NL.

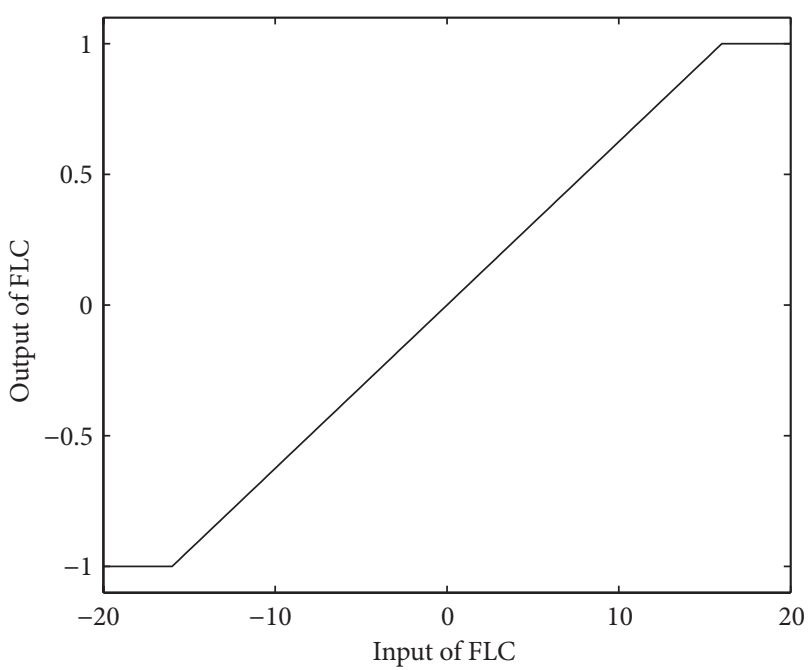

FIGURE 7: Input-output relation of the FLC.

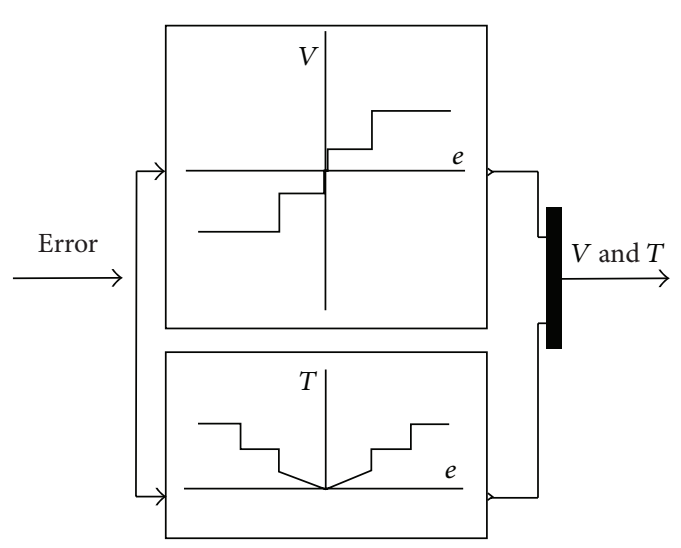

Figure 8: Typical functions for $V$ and $T$.

Product inference is applied for fuzzy inference and the center of gravity method is adopted for defuzzification. The input-output (error-rotation angel) relation of the proposed controller is shown in Figure 7. The rotation angel of a DC motor is proportional to two variables. One is the time $T$ during which voltage is fed to motor, and the other is the level of this voltage, $V$. In other words, control command is a combination of $T$ and $V$. Figure 8 shows functions $V(e)$ and $T(e)$ that are obtained from heuristics to satisfy the relation shown in Figure 7. The control commands emitted by MAT$\mathrm{LAB}$ program are fed to motors through a microcontroller.

Note that LDR-based mode of tracking is, also, active if the sun spot is not detected on the transparent screen. Furthermore, energy saving mode has been considered active when the solar radiation is not great enough for the system to produce electricity. In these conditions, the system has been hold at the last known position for some minutes, and then the tracking algorithm will start again. Figure 9 illustrates the flow chart of the system operation. 


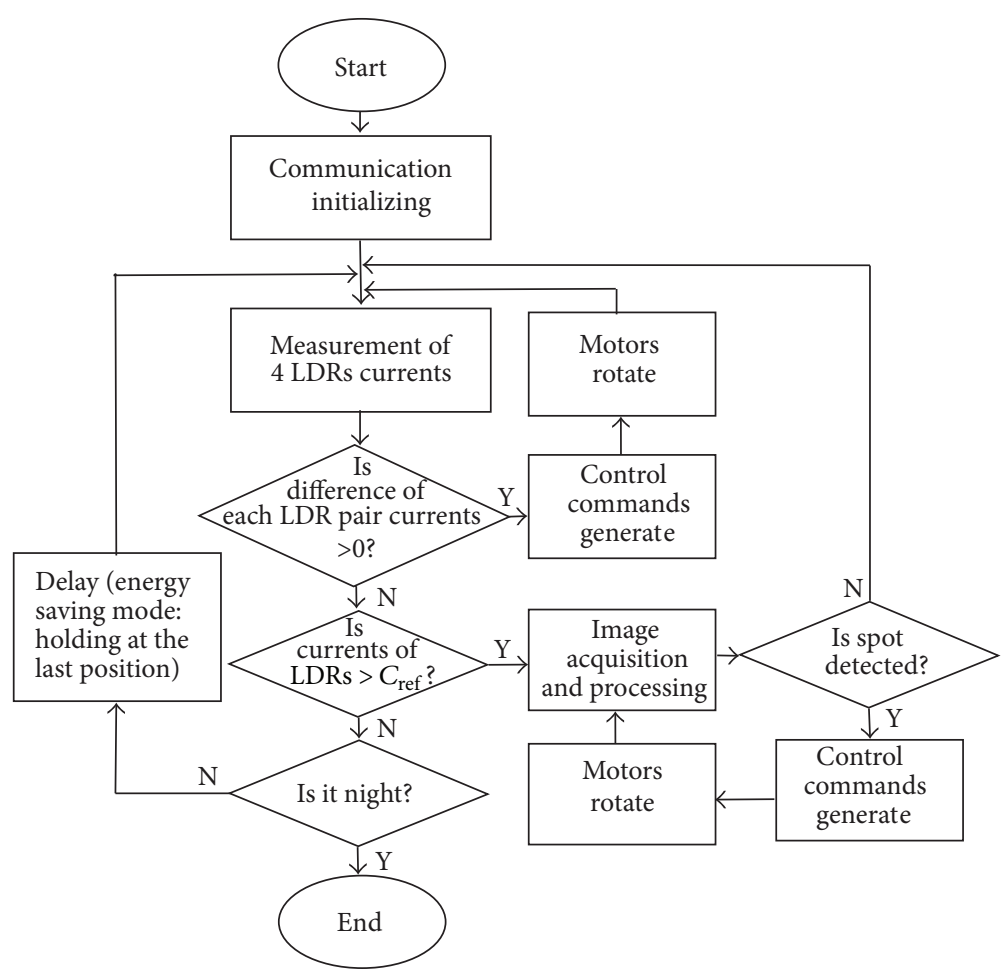

FIGURE 9: Flowchart of the system operation.

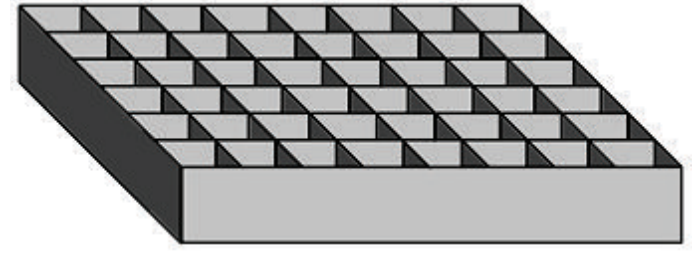

FIGURE 10: Schematic of netted plate installed on the PV panels.

\section{Experiments and Results}

The performance of the designed DAST has been evaluated and monitored during several days on September and October 2012. Since flat PV solar panels were only available, several sheets of aluminum as a netted plate were mounted on the two 5 watts amorphous type PV panels perpendicularly (Figure 10). This netted plate guarantees that no solar radiation gets to the PV panel when the tracking error is greater than some small degrees, so, the results of the proposed installation is logically close to a CPV solar panel. The used PV panels parameters are provided in Table 1.

Figure 11 shows the experimental power (the product of the open-circuit voltage and short-circuit current) attained using the proposed tracking system, as well as the one obtained from fixed panel, on October 5. The output power showed a considerable increase during the early and late hours of the day. In fact, the average improvement, in the tracking system, was about $81 \%$ and $105 \%$ in the morning (7:30-9:00) and in the afternoon (15:00-17:00), respectively.

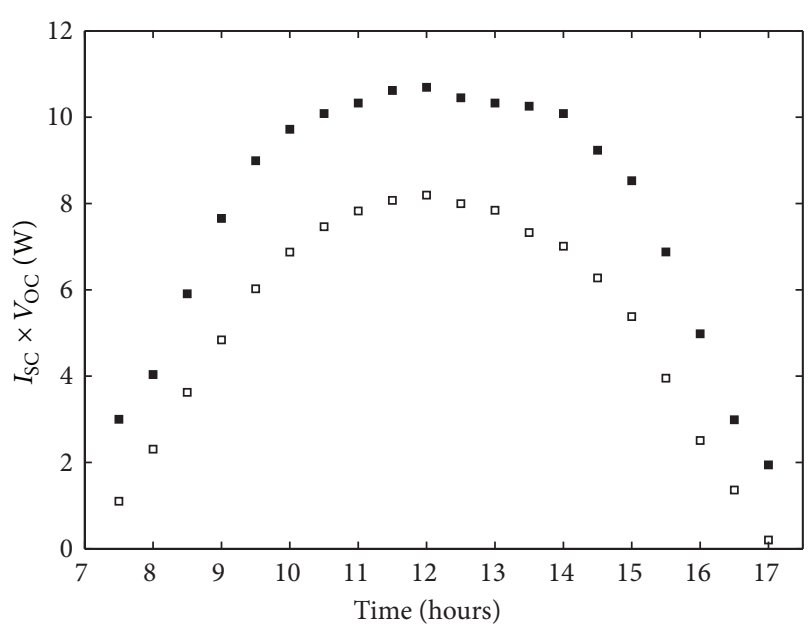

- With sun tracking

- Without sun tracking

FIGURE 11: The power generation comparison of the fixed angle and tracking systems (experiment on October 5).

However, the improvement was about $30-41 \%$ during midday. The mean power generated by the sun tracker panel during the whole day was $60.45 \%$ higher than the fixed one. Figure 12 illustrates absolute and relative increases in generated power using sun tracker.

In Figure 13, the coordinates of the center of the sun spot are shown for a period of time at the experiment day. It can be seen in this figure that the strategy based on image processing 
TABLE 1: Solar cell description.

\begin{tabular}{lc}
\hline Parameter & Value \\
\hline Maximum power $\left(P_{m}\right)$ & $5(\mathrm{~W})$ \\
Voltage at $P_{m}$ & $18(\mathrm{~V})$ \\
Current at $P_{m}$ & $277(\mathrm{~mA})$ \\
Open circuit voltage & $27(\mathrm{~V})$ \\
Short circuit current & $346(\mathrm{~mA})$ \\
\hline
\end{tabular}

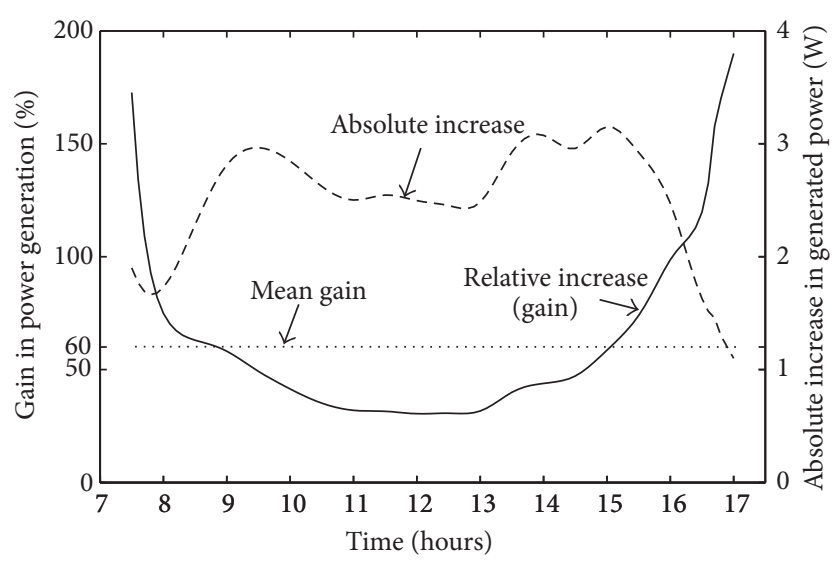

FIGURE 12: Power generation improvement obtained by using the proposed system (experiment on October 5).

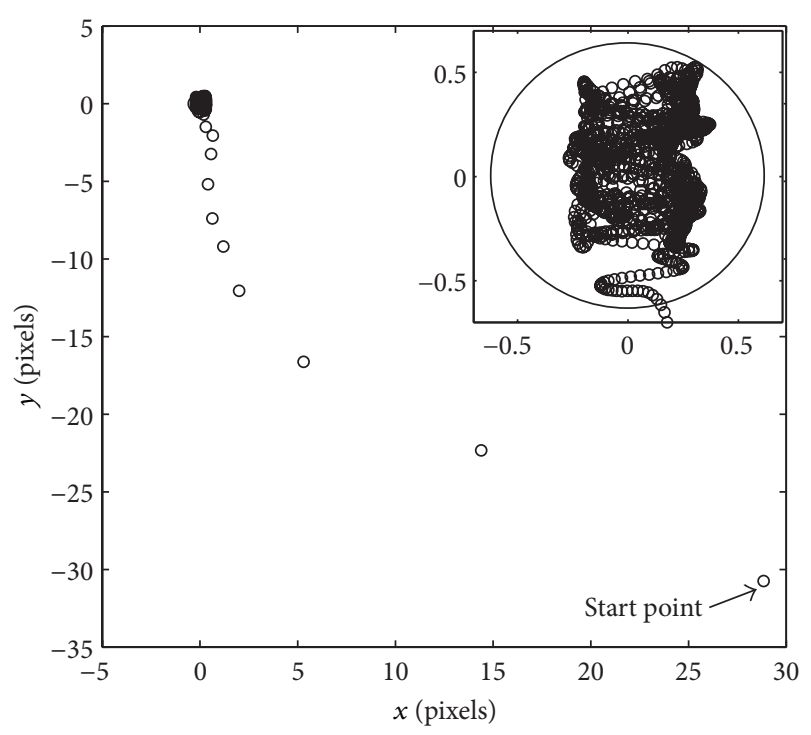

FIGURE 13: Tracking error (experiment on October 5).

pushed the center of the sun spot into a circle with a radius of 0.6 pixels. This value is corresponding to $0.1432^{\circ}$ of the sun rays incident angel, according to the webcam resolution and geometry of the imaging devise. So, tracking error is less than $0.15^{\circ}$. An open-loop solar tracker that provides a tracking error in the range from $0.236^{\circ}$ to $0.693^{\circ}$ is presented in [8]. So, the closed-loop system presented in this work has higher precision than the open-loop one.

\section{Conclusion}

A new sun tracking system that provides high tracking precision, needed by concentration PVs, has been developed using fuzzy logic and image processing. The system consists of two main modes. One is LDR-based that adjusts the orientation of the solar panel approximately, and the other is based on image processing that provides small tracking errors (less than 0.15 degrees). Energy saving mode has been considered also, to prevent power overconsumption during cloudy skies. Experimental results showed the significant increase in the power generated by the solar panel. The proposed system has low cost and is independent of correct initial configuration and geographical position. It can be used with various collectors and solar concentrators, as well as in astronomical researches as a leading system for telescopes.

\section{Conflict of Interests}

The authors declare that there is no conflict of interests regarding the publication of this paper.

\section{References}

[1] F. R. Rubio, M. G. Ortega, F. Gordillo, and M. López-Martínez, "Application of new control strategy for sun tracking," Energy Conversion and Management, vol. 48, no. 7, pp. 2174-2184, 2007.

[2] N. H. Helwa, A. B. G. Bahgat, A. M. R. El Shafee, and E. T. El Shenawy, "Maximum collectable solar energy by different solar tracking systems," Energy Sources, vol. 22, no. 1, pp. 23-34, 2000.

[3] C. S. Chin, A. Babu, and W. McBride, "Design, modeling and testing of a standalone single axis active solar tracker using MATLAB/Simulink," Renewable Energy, vol. 36, no. 11, pp. 3075-3090, 2011.

[4] S. Ozcelik, H. Parkash, and R. Challoo, "Two-axis solar tracker analysis and control for maximum power generation," Procedia Computer Science, vol. 6, pp. 457-462, 2011.

[5] H. Arbab, B. Jazi, and M. Rezagholizadeh, "A computer tracking system of solar dish with two-axis degree freedoms based on picture processing of bar shadow," Renewable Energy, vol. 34, no. 4, pp. 1114-1118, 2009.

[6] M. J. Clifford and D. Eastwood, "Design of a novel passive solar tracker," Solar Energy, vol. 77, no. 3, pp. 269-280, 2004.

[7] R. Ranganathan, W. Mikhael, N. Kutkut, and I. Batarseh, "Adaptive sun tracking algorithm for incident energy maximization and efficiency improvement of PV panels," Renewable Energy, vol. 36, no. 10, pp. 2623-2626, 2011.

[8] K. K. Chong and C. W. Wong, "General formula for on-axis sun-tracking system and its application in improving tracking accuracy of solar collector," Solar Energy, vol. 83, no. 3, pp. 298305, 2009.

[9] W. Batayneh, A. Owais, and M. Nairoukh, "An intelligent fuzzy based tracking controller for a dual-axis solar PV system," Automation in Construction, vol. 29, pp. 100-106, 2013.

[10] C.-Y. Lee, P.-C. Chou, C.-M. Chiang, and C.-F. Lin, "Sun tracking systems: a review," Sensors, vol. 9, no. 5, pp. 3875-3890, 2009.

[11] H. Mousazadeh, A. Keyhani, A. Javadi, H. Mobli, K. Abrinia, and A. Sharifi, "A review of principle and sun-tracking methods for maximizing solar systems output," Renewable and Sustainable Energy Reviews, vol. 13, no. 8, pp. 1800-1818, 2009. 
[12] J. Cañada, M. P. Utrillas, J. A. Martinez-Lozano, R. Pedrós, J. L. Gómez-Amo, and A. Maj, "Design of a sun tracker for the automatic measurement of spectral irradiance and construction of an irradiance database in the $330-1100 \mathrm{~nm}$ range," Renewable Energy, vol. 32, no. 12, pp. 2053-2068, 2007.

[13] M. Alata, M. A. Al-Nimr, and Y. Qaroush, "Developing a multipurpose sun tracking system using fuzzy control," Energy Conversion and Management, vol. 46, no. 7-8, pp. 1229-1245, 2005.

[14] I. Luque-Heredia, J. M. Moreno, P. H. Magalhães, R. Cervantes, G. Quéméré, and O. Laurent, "Inspira's CPV sun tracking," Springer Series in Optical Sciences, vol. 130, pp. 221-251, 2007.

[15] K. Aiuchi, K. Yoshida, M. Onozaki, Y. Katayama, M. Nakamura, and K. Nakamura, "Sensor-controlled heliostat with an equatorial mount," Solar Energy, vol. 80, no. 9, pp. 1089-1097, 2006.

[16] M. Abouzeid, "Use of a reluctance stepper motor for solar tracking based on a programmable logic array (PLA) controller," Renewable Energy, vol. 23, no. 3-4, pp. 551-560, 2001.

[17] S. Gagliano and N. Savalli, "Two-axis sun tracking system: design and simulation," Eurosun 2006, 2006.

[18] E. T. El Shenawy, M. Kamal, and M.A. Mohamad, "Artificial intelligent control of solar tracking system," Journal of Applied Sciences Research, vol. 8, pp. 3971-3984, 2012. 

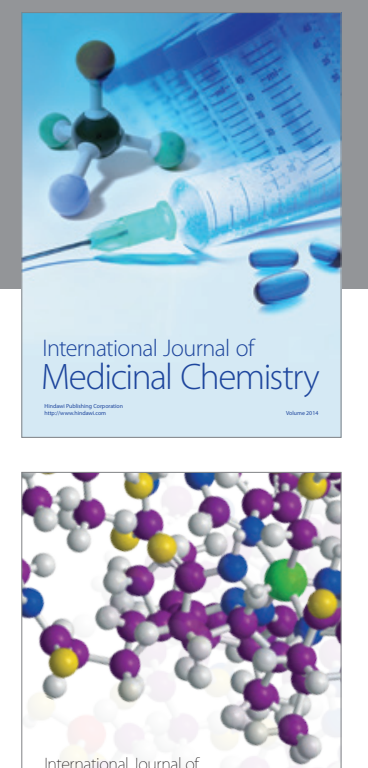

\section{Carbohydrate} Chemistry

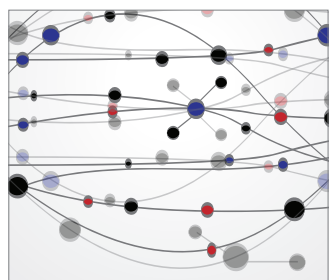

The Scientific World Journal
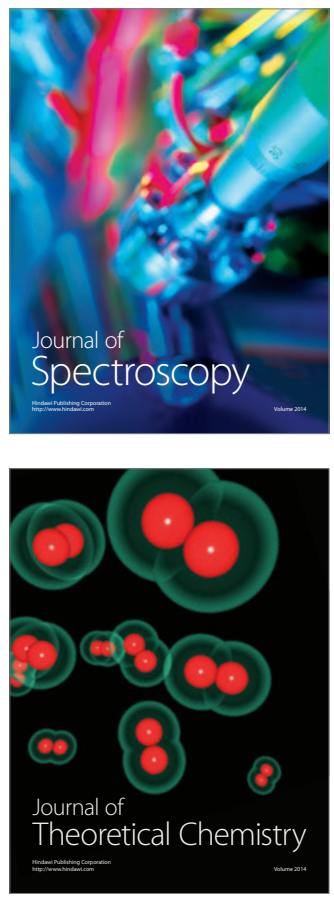
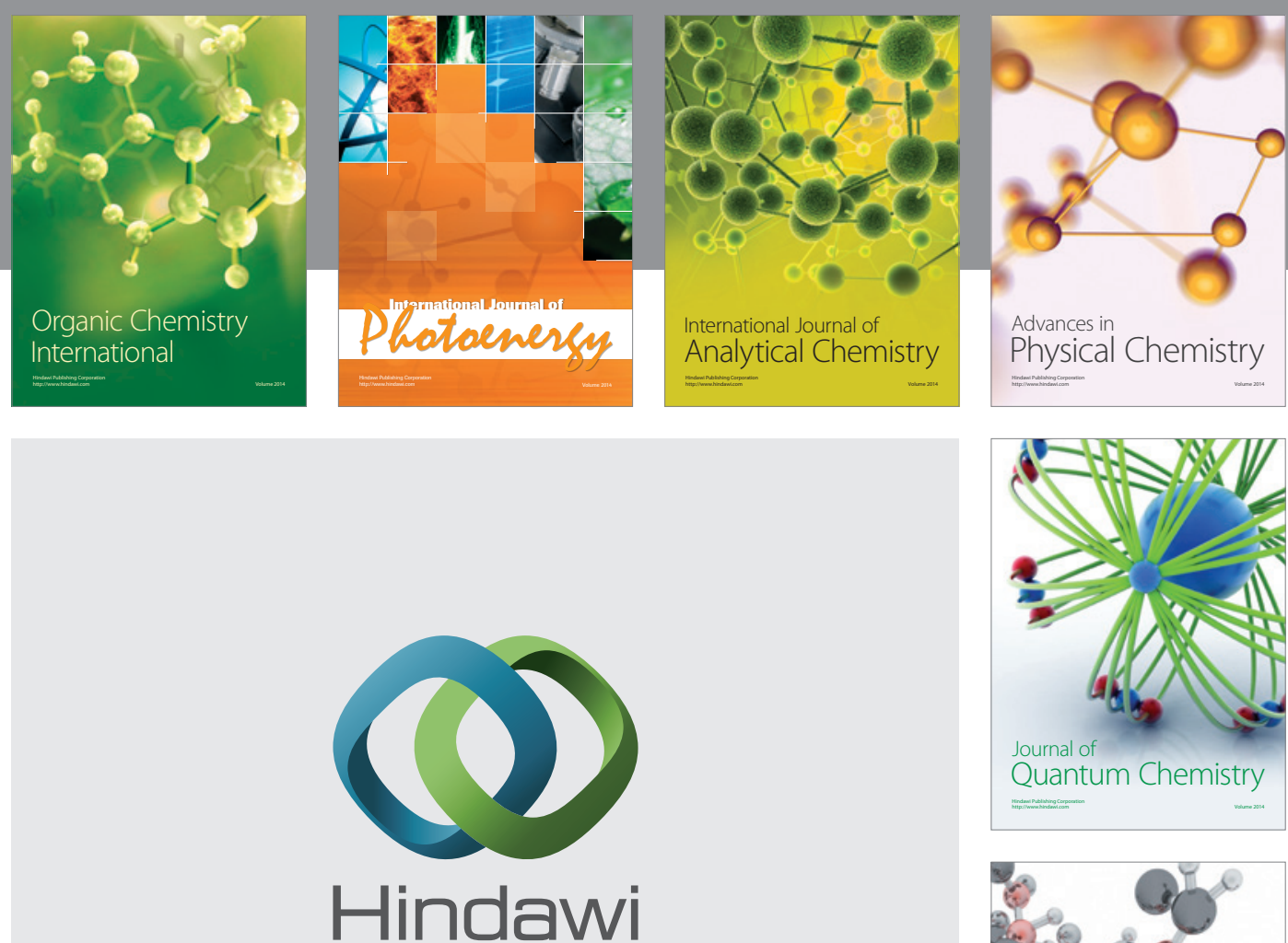

Submit your manuscripts at

http://www.hindawi.com

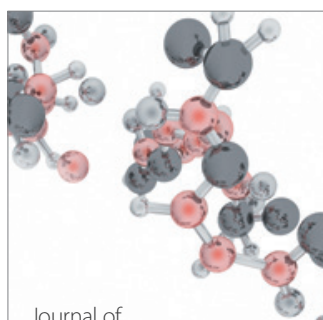

Analytical Methods

in Chemistry

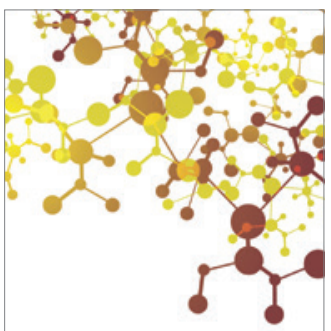

Journal of

Applied Chemistry

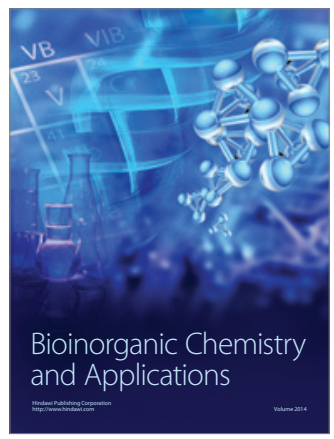

Inorganic Chemistry
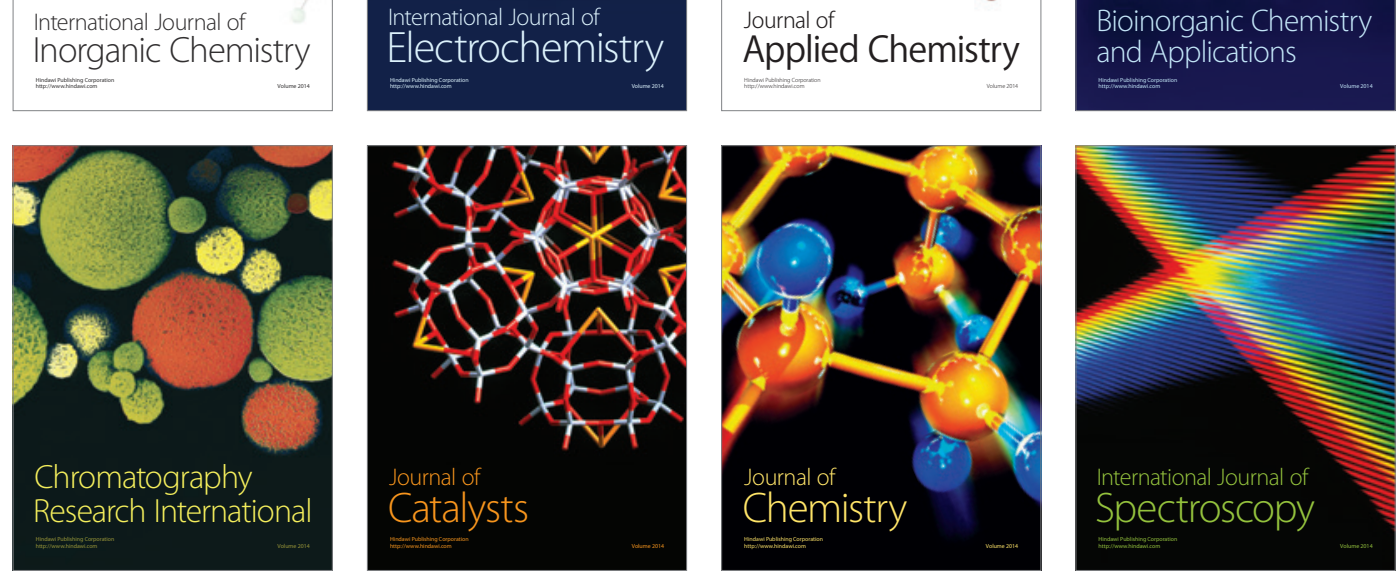